\title{
Phase II study of capecitabine as palliative treatment for patients with recurrent and metastatic squamous head and neck cancer after previous platinum-based treatment
}

\author{
J Martinez-Trufero*, , D Isla², JC Adansa ${ }^{3}$, A Irigoyen ${ }^{4}$, R Hitt ${ }^{5}$, I Gil-Arnaiz', J Lambea ${ }^{2}$, MJ Lecumberri' and \\ JJ Cruz ${ }^{3}$ on behalf of the Spanish Head and Neck Cancer Treatment Group (TTCC) \\ 'Medical Oncology Department of University Hospital Miguel Servet Hospital, Paseo de Isabel La Católica 1-3, 50009 Zaragoza, Spain; ${ }^{2}$ Medical \\ Oncology Department of University Hospital Lozano Blesa Hospital, Avda. San Juan Bosco, 1 5. 50009 Zaragoza, Spain; ${ }^{3}$ Medical Oncology Department \\ of University Hospital Clínico de Salamanca Hospital, Paseo de San Vicente 58-I 82, 37007 Salamanca, Spain; ${ }^{4}$ Medical Oncology Department of \\ University Hospital Virgen de las Nieves Hospital, Avda de las Fuerzas Armadas, 2, 18014 Granada, Spain; ${ }^{5}$ Medical Oncology Department of University \\ Hospital 12 de Octubre Hospital, Avda. de Córdoba s/n, 2804I Madrid, Spain
}

\begin{abstract}
BACKGROUND: Platinum-based therapy (PBT) is the standard therapy for recurrent and/or metastatic head and neck cancer (HNC), but the incidence of recurrence remains high. This study evaluates the efficacy and tolerability of capecitabine as palliative monotherapy for recurrent HNC previously treated with PBT.

METHODS: Patients aged 18-75 years, with Eastern Cooperative Oncology Group performance status 0-2, squamous HNC with locoregional and/or metastatic recurrence previously treated with PBT and adequate organ functions, were included. Capecitabine ( $1.250 \mathrm{mg} \mathrm{m}^{-2}$ BID) was administered on days I - 14 every 21 days for at least two cycles.

RESULTS: A total of 40 male patients with a median age of 58 years were analysed. All patients received a median number of four cycles of capecitabine (range: I -9) and the median relative dose intensity was $91 \%$. Seven patients were not evaluable for response. Overall response rate was $24.2 \%$. Median time to progression and overall survival were 4.8 and 7.3 months, respectively. Haematological adverse events (AEs) grade $3 / 4$ were reported in six patients. Most common grade $3 / 4$ non-haematological AEs were asthenia (12.5\%), palmar-plantar eritrodisestesia (10\%), mucositis (10\%), dysphagia (10\%) and diarrhoea (7.5\%).

CONCLUSIONS: Capecitabine seems to be an active, feasible and well-tolerated mode of palliative treatment for advanced HNC patients who have previously received PBT schedules.

British Journal of Cancer (2010) I 02, I687-1691. doi:I0.1038/sj.bjc.6605697 www.bjcancer.com
\end{abstract}

Published online 18 May 2010

(c) 2010 Cancer Research UK

Keywords: head and neck cancer; capecitabine; platinum pretreated; advanced disease

Treatment of recurrent or metastatic head and neck cancer (HNC) is a frequent problem in daily clinical practice. Between 20 and $40 \%$ of patients with stage I or II and $>70 \%$ of patients with stage III or IV at initial diagnosis, which are treated with curative intention, will recur (Vokes et al, 1993). Overall, locoregional recurrence is highly common and the major cause of death in $40-60 \%$ of these patients, whereas $<20 \%$ die because of distant metastasis.

In common practice, chemotherapy (CT) is considered as the first-line treatment in the majority of patients with recurrent and/or metastatic HNC, with platinum-based therapy (PBT) being the main alternative to treat symptoms (Hong and Bromer, 1983). Recently, the introduction of epidermal growth factor receptor (EGFR) inhibitors, such as cetuximab, together with PBT, has improved response and survival rates in these patients (Vermorken et al, 2008). The progressive introduction of multimodal treatments in which CT is used as part of an initial treatment, either as neoadjuvant or concomitantly with radio-

* Correspondence: Dr J Martinez-Trufero; E-mail: jmtrufero@seom.org Received 5 March 20 I0; revised 21 April 20I0; accepted 21 April 20 10; published online 18 May 2010 therapy, results in an increasing number of patients presenting recurrence or distant progression, who have already been treated with platinum derivates previously (Pignon et al, 2000). In addition, the majority of patients in this situation report disease progression in previously radiated areas in which the possibility of response notably diminishes. The clinical condition for these patients is not always the optimal to receive excessively aggressive treatments; therefore in the palliative context, the use of effective and well-tolerated treatments is required.

Capecitabine (Xeloda; Hoffmann-La Roche Inc., Nutley, NJ, USA) is an oral fluoropyrimidine prodrug, which is absorbed in the gastrointestinal tract leading to 5-fluorouracil (5FU) plasma levels, which are superior to those achieved through 5FU intravenous infusion but with an improved safety profile because of its selective tumour activation. Moreover, oral administration offers the obvious advantage of convenience for patients. Capecitabine use has been extensively studied and has been approved in colorectal and breast cancer.

The purpose of this phase II study was to assess the efficacy and safety of capecitabine when used as palliative monotherapy for recurrent and/or metastatic HNC previously treated with PBT. 


\section{PATIENTS AND METHODS}

\section{Eligibility}

Patients with recurrent or metastatic squamous HNC who were considered ineligible for curative surgery or chemoradiotherapy were enrolled at selected centres belonging to the Spanish HNC treatment Group (TTCC). Main eligibility criteria were measurable disease as defined by Response Evaluation Criteria in Solid Tumours (RECIST) (Therasse et al, 2000); treatment at least once with PBT as initial treatment or as first-line treatment for recurrent/metastatic disease. No more than one previous systemic therapy and no CT or radiotherapy within 4 weeks of study entry was allowed. Other eligibility criteria included the following: age 18-75 years; a negative result in pregnancy test; life expectancy of $\geqslant 3$ months; an Eastern Cooperative Oncology Group (ECOG) performance status of $\leqslant 2$; and adequate organ and marrow function defined as a leukocyte count $\geqslant 3000$ per $\mu \mathrm{l}$, an absolute neutrophil count $\geqslant 1500$ per $\mu \mathrm{l}$, a platelet count $\geqslant 100000$ per $\mu \mathrm{l}$, a total bilirubin within normal institutional limits, plasma AST and ALT levels $\leqslant 2.5$ times the institutional upper limit of normal and a creatinine level $\leqslant 1.5 \mathrm{mg}$ per $100 \mathrm{ml}$; no uncontrolled serious concomitant disease; no cavum lymphoepithelioma; and no CNS metastasis.

The study was conducted after approval by an Independent Ethics Committee of each site and in accordance with the Declaration of Helsinki, Good Clinical Practices, and local ethical and legal requirements. Signed informed consent was obtained from all patients before study entry.

\section{Treatment plan and dose modifications}

Oral capecitabine was administered at an initial dose of $1.250 \mathrm{mg} \mathrm{m}^{-2}$ BID for 1-14 days, followed by a rest period of 1 week, every 21 days. Administration of capecitabine dissolved in water or through a feeding tube was allowed for patients unable to swallow tablets. Treatment was administered for at least three cycles and was continued until disease progression, intercurrent illness preventing further administration, unacceptable toxicity or patient decision. Patients were assessed for toxicity before each cycle using the NCI-CTCAE (National Cancer Institute common toxicity criteria) version 2.0. Patients experiencing unacceptable grade 2 adverse events (AEs) had therapy temporarily withheld until resolution to grade 1 or less. If, on restarting therapy, $\mathrm{AE}$ continued, the dose was decreased to $75 \%$ of the dose. Any grade 3 or $4 \mathrm{AE}$ required temporary discontinuation of therapy until resolution to grade 1 or less and restarted at $50 \%$ of the dose. Treatment was definitively interrupted in patients whose AE did not resolve after 2 weeks of discontinuation or who required a second dose reduction. Once a patient's dose was reduced, it was not subsequently increased.

\section{Response assessments}

Patients were re-evaluated clinically, at every cycle, and radiographically every three cycles. The same tumour assessment technique was used throughout the study. Response guidelines as defined by RECIST v1.0 criteria were used (Therasse et al, 2000). Tumour response was evaluated after three cycles of treatment as either a complete response (CR), partial response (PR), progressive disease (PD) or stable disease (SD). Disease control was defined as the sum of patients achieving a CR, PR or SD. Confirmation of all responses was required after a minimum of 4 weeks. After completion of study treatment, patients with SD or objective response were followed up every 3 months until $\mathrm{PD}$ and/or death. Patients were evaluated for AEs during therapy and until 28 days after the last study drug dose.

\section{Statistical analysis}

The study used a two-stage design requiring the enrolment of 17 patients in the first stage. The study would be stopped if less than two responses were observed at the end of the first stage. If more than two responses were observed, an additional number of patients were to be enrolled in the second stage to achieve the minimum foreseen 35 evaluable patients. This design provided an $\alpha$ level of $5 \%$ and a $\beta$ level of $10 \%$. The primary end point was response rate. Secondary end points included time to progression (TTP), overall survival (OS) and toxicity. All patients who met the eligibility criteria and received at least three cycles of treatment were evaluable for response and were included in the efficacy analysis. All enrolled patients who received the study drug were included in the toxicity analysis.

Both TTP and OS were measured from the date of enrolment until disease progression or death, respectively, and were summarised by Kaplan-Meier curves. Factors related to response or lack of early progression were analysed using Fisher's exact test, and factors related to survival were analysed using the log-rank test and the Cox proportional hazards model. All statistical analyses were conducted at a level of significance of $95 \%$ $(\alpha=0.05)$.

\section{RESULTS}

\section{Patients and eligibility}

From October 2005 to July 2008, 40 patients with relapsed oropharyngeal, larynx and oral cavity HNC were enrolled into the study at five sites in Spain. Their characteristics are summarised in Table 1. Median time from initial diagnosis until inclusion in study was 17.7 months (range: $7.2-105.7$ ).

As part of initial radical treatment, 25 patients $(62.5 \%)$ had received neoadjuvant $\mathrm{PBT}$ and 26 patients (65\%) had received radiotherapy + PBT concomitantly. In all, 19 patients $(47.5 \%)$ had undergone surgery previously. Seven patients (17.5\%) had received postoperative radiotherapy, four of them with concomitant cisplatin. Median time from surgery until inclusion in the study was 22.1 months (range: 3.1-52.1). Median time from diagnosis of recurrent/metastatic disease until inclusion in the study was 1.1 months (range: $0-52.1$ ). Six patients $(15 \%)$ had received PBT as part of first-line treatment in recurrent/metastatic disease.

Median follow-up for all patients was 6.6 months (range: $0.6-35)$. Median follow-up for surviving patients was 11.5 months $(2.7-35)$. In total, 16 patients $(40 \%)$ received subsequent rescue therapy. In seven of them, second-line therapy was administered after disease progression and mainly consisted of cetuximab-based $(n=2)$, metotrexate $(n=2)$, paclitaxel plus carboplatin $(n=1)$ and other $(n=5)$ chemotherapeutic regimens.

\section{Treatment exposure}

All patients received a total of 182 cycles of capecitabine with a median of 4 cycles per patient (range: 1-9).

Seven patients were not evaluable for response as they do not receive at least three cycles: three died (two because of unknown reasons and one because of an infection), two patients because of AEs and two because of a decrease in ECOG performance status. All these patients were included in the toxicity analysis.

Capecitabine dose reduction was necessary in seven $(3.8 \%)$ cycles because of non-haematological (four cycles) or haematological (one cycle) AEs. A total of 33 (18.1\%) cycles were delayed, 10 were delayed for non-haematological AEs, 2 for haematological AEs, 1 because of the presence of both toxicities and 20 for non-drug-related reasons. The median relative dose intensity was equivalent to $91 \%$ of the predicted dose intensity. 
Table I Patient demographics $(N=40)$

\begin{tabular}{|c|c|c|}
\hline \multirow[b]{2}{*}{ Characteristics } & \multicolumn{2}{|c|}{ Patients } \\
\hline & No. & $\%$ \\
\hline \multicolumn{3}{|l|}{ Gender } \\
\hline Men & 40 & 100 \\
\hline \multicolumn{3}{|l|}{ Age (years) } \\
\hline Mean (range) & \multicolumn{2}{|c|}{$58.4(40.7-75.1)$} \\
\hline \multicolumn{3}{|l|}{ Performance status (ECOG) } \\
\hline 0 & 10 & 25 \\
\hline I & 28 & 70 \\
\hline 2 & 2 & 5 \\
\hline \multicolumn{3}{|l|}{ Disease status at enrolment } \\
\hline Local disease recurrence & 33 & 82.5 \\
\hline Locoregional disease & 7 & 17.5 \\
\hline Distant metastases & 20 & 50 \\
\hline Lung & 12 & \\
\hline Other & 8 & \\
\hline Neoadjuvant therapy & 25 & 62.5 \\
\hline Taxane-cisplatin-5FU triplets & 18 & \\
\hline Cisplatin-5FU & 6 & \\
\hline Cisplatin-docetaxel & । & \\
\hline Surgery & 19 & 47.5 \\
\hline Primary tumour & 7 & \\
\hline Nodal disease & 3 & \\
\hline Primary tumour and nodal disease & 8 & \\
\hline Lung node metastases & I & \\
\hline \multicolumn{3}{|l|}{ Adjuvant therapy } \\
\hline RT alone & 29 & 72.5 \\
\hline $\mathrm{RT}+\mathrm{CT}$ with & 26 & 65.0 \\
\hline Cisplatin & 21 & \\
\hline Taxane-cisplatin-5FU triplets & 4 & \\
\hline Cisplatin-docetaxel & I & \\
\hline Postoperative RT alone & 7 & 17.5 \\
\hline Postoperative $\mathrm{RT}+\mathrm{CT}$ with cisplatin & 4 & \\
\hline \multicolumn{3}{|l|}{ Recurrent/metastatic disease treatment } \\
\hline CT & 6 & 15 \\
\hline Cisplatin-5FU & 2 & \\
\hline Carboplatin-paclitaxel & 2 & \\
\hline Cisplatin & I & \\
\hline Taxane-cisplatin-5FU triplets & I & \\
\hline RT & 3 & 7.5 \\
\hline Surgery & 11 & 27.5 \\
\hline
\end{tabular}

Reasons for discontinuation of treatment were as follows: disease progression in 12 patients $(30 \%)$, investigator criteria in $13(32.5 \%)$, AEs in $8(20 \%)$ patients and death in 7 patients $(17.5 \%)$.

\section{Treatment responses}

Two patients had PR after 3 cycles among the 17 patients entered during the first stage; thus, from this standpoint, the drug was declared sufficiently active to warrant further study in the second stage. Of the total 40 patients enrolled, only 33 were evaluable (as they received $\geqslant 3$ cycles of treatment) with two CR and 6 PRs for an overall response rate of $24.2 \%$ (95\% confidence interval $(95 \%$ CI): $9.6-38.9 \%)$. A total of 18 patients (54.5\%) had SD as their best response. Therefore, as defined above, $79 \%$ of patients experienced

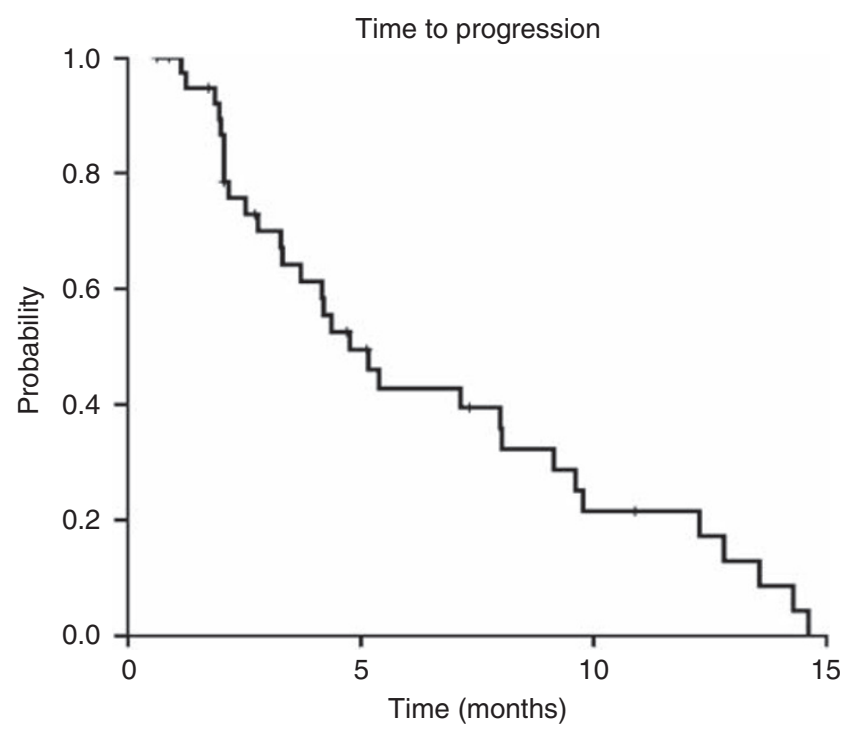

Figure I Time to progression.

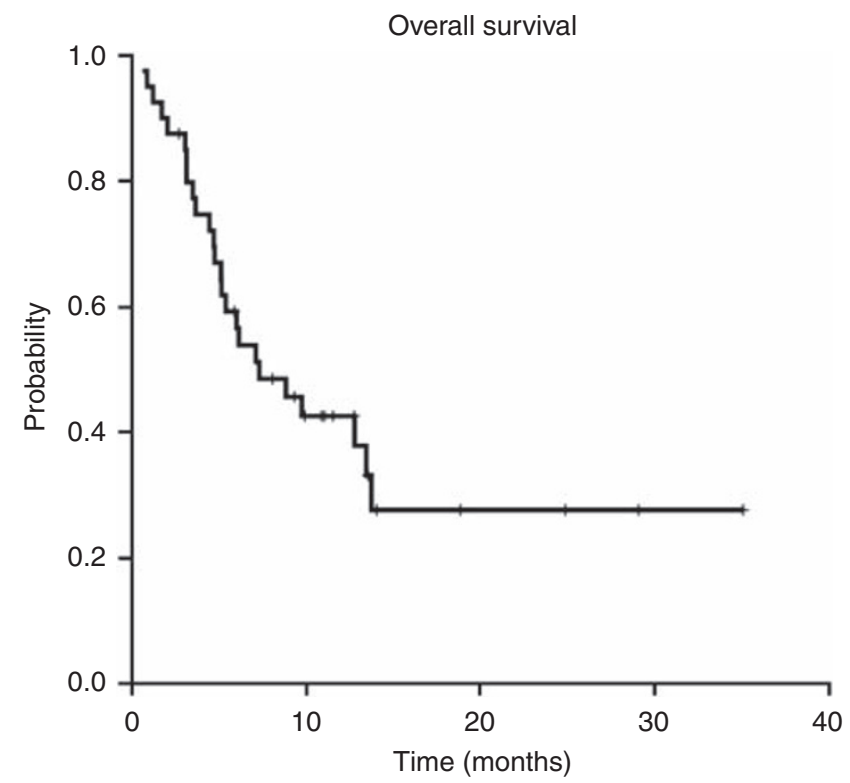

Figure 2 Overall survival.

some degree of disease control. The remaining seven patients $(21.2 \%)$ had PD at initial re-evaluation. The median duration of response was 8.4 months (range: $3.3-13.5$ months).

\section{TTP and OS}

The median TTP was 4.8 months (95\% CI: 3.2-6.3 months; Figure 1). Median OS was 7.3 months (95\% CI: 3.0 - 11.6 months; Figure 2) for the entire cohort, with a survival probability of $42 \%$ per year. In all, 15 of the 33 assessable patients continue to be alive.

\section{Toxicity}

All 40 patients were included in the toxicity analysis as they received at least one cycle of treatment. A total of 37 patients (92.5\%) reported treatment-related AEs. A summary of the most common treatment-related AEs per patient is shown in Table 2. The most common were haematological (anaemia), dermatological 
Table 2 Most common (>5\%) AEs per patient according to $\mathrm{NCl}-\mathrm{CTC}$ grade $(n=40)$

\begin{tabular}{|c|c|c|c|}
\hline \multirow[b]{3}{*}{ NCI-CTCAE toxicity } & \multicolumn{3}{|c|}{ No. patients (\%) } \\
\hline & \multirow[b]{2}{*}{ Grade I/Grade2 } & \multicolumn{2}{|c|}{ Overall $(n=40)$} \\
\hline & & Grade 3 & Grade 4 \\
\hline \multicolumn{4}{|l|}{ Haematological events } \\
\hline Anaemia & $24(60)$ & $2(5.0)$ & - \\
\hline Leucopoenia & $6(15)$ & I (2.5) & - \\
\hline Neutropaenia & $4(10)$ & I (2.5) & - \\
\hline Low platelet count & $3(7.5)$ & - & I (2.5) \\
\hline \multicolumn{4}{|l|}{ Non-haematological events } \\
\hline Palmar-plantar eritrodisestesia & II (27.5) & $3(7.5)$ & I (2.5) \\
\hline Mucositis & $10(25)$ & $4(10.0)$ & - \\
\hline Asthenia & $8(20)$ & $5(12.5)$ & - \\
\hline Dysphagia & $4(10.0)$ & $3(7.5)$ & I (2.5) \\
\hline Diarrhoea & $3(7.5)$ & $3(7.5)$ & - \\
\hline Anorexia & $5(12.5)$ & I (2.5) & - \\
\hline Vomiting & $4(10.0)$ & I (2.5) & - \\
\hline Weight loss & $4(10.0)$ & I (2.5) & - \\
\hline Bilirubin & $5(12.5)$ & - & - \\
\hline Skin rash/desquamation & $4(10)$ & - & - \\
\hline AST & $4(10)$ & - & - \\
\hline Infection without neutropaenia & I (2.5) & $2(5.0)$ & - \\
\hline Nausea & $3(7.5)$ & - & - \\
\hline
\end{tabular}

Abbreviations: $\quad \mathrm{AE}=$ adverse event; $\mathrm{AST}=$ aspartate transaminase; $\mathrm{NCl}$

$\mathrm{CTCAE}=$ National Cancer Institute common toxicity criteria.

(palmar-plantar eritrodisestesia) and gastrointestinal (mucositis and asthenia).

Grade 3/4 haematological treatment-related AEs were reported in six patients. The most frequently observed grade $3 / 4$ non-haematological AEs by patient were asthenia (12.5\%), palmar-plantar eritrodisestesia (10\%), mucositis (10\%), dysphagia $(10 \%)$ and diarrhoea $(7.5 \%)$.

Eight $(20 \%)$ patients discontinued treatment because of AEs. The main reasons for discontinuation were grade $2 / 3$ palmarplantar eritrodisestesia, grade 3 asthenia, grade 3 mucositis, grade 3 diarrhoea and grade 3 vomiting.

Seven patients $(17.5 \%)$ died during the study treatment, five died within the first 60 days of the study because of PD (one patient), unknown reasons (two patients), opioid intoxication (one patient) and serious respiratory infection with pleural effusion and cardiac failure (one patient).

\section{DISCUSSION}

To date, cisplatin, methotrexate, 5FU, paclitaxel and docetaxel (Catimel et al, 1994; Forastiere et al, 1998) have been the most widely used drugs for advanced or recurrent disease, with variable response rates ranging from 15 to $40 \%$. The use of polychemotherapy mainly based in cisplatin-5FU combinations would result in better response rates, but no improvement in OS has been noted when compared with monotherapy, and the combination has been associated with a substantial toxicity risk that is unacceptable in the palliative setting (Forastiere et al, 1992). In the last year, the introduction of EGFR inhibitors, such as cetuximab, added to conventional CT has been shown to be an efficacious instrument to improve the response rate, progressionfree survival (PFS) and OS (Vermorken et al, 2008).

The activity of capecitabine in HNC was first reported in 2002 after the results of the first phase I studies with capecitabine in combination with cisplatin or radiotherapy in $\mathrm{HNC}$
(Arnedos et al, 2002; Pivot et al, 2003; Sykes et al, 2004). These findings have been supported by a recent phase I study on the use of capecitabine in combination with intensity-modulated radiation therapy (Thomas et al, 2009). The maximal tolerable dose of capecitabine monotherapy for phase II studies was established in these phase I studies.

Several phase II studies have been reported with capecitabine in combination with radiotherapy and/or CT in advanced or recurrent HNC. Two phase II studies have been reported with capecitabine (administered at doses of $825 \mathrm{mg} \mathrm{m}^{-2}$ (Kim et al, 2005) and $2000 \mathrm{mg} \mathrm{m}^{-2}$ (Hitt et al, 2004) on days 1-14) in combination with cisplatin (doses of $75-80 \mathrm{mg} \mathrm{m}^{-2}$ on day 1) every 21 days. Kim et al (2005) studied the capecitabine/cisplatin combination in 37 patients with locally advanced stage. The results observed (CR of $78.4 \%$, PR of $16.2 \%$ and 2 -year OS of $57.9 \%$ ) were comparable with those reported in most chemoradiotherapy studies. Hitt et al (2004) evaluated the capecitabine/cisplatin combination in 41 patients with recurrent and/or metastatic squamous HNC, observing an ORR of $68 \%$ (RC 39\%, RP 29\%). Only five patients (12\%) had received PBT. Median PFS was 6.4 months and median OS was 12.6 months. Another phase II (Bentzen and Hansen, 2007) assessed the use of capecitabine (825 $\mathrm{mg} \mathrm{m}^{-2}$ on days $1-14$ every 21 days) in combination with paclitaxel $\left(175 \mathrm{mg} \mathrm{m}^{-2}\right)$ every 21 days in 50 patients with recurrent and/or metastatic HNC, observing a response rate of $42 \%$ ( $4 \% \mathrm{CR}$, $38 \%$ PR, $28 \%$ NC) and a median OS of 8 months with an acceptable toxicity (only $10 \%$ of patients required modifications of the foreseen dose).

In addition, one phase II study has assessed the efficacy of capecitabine as single agent in 17 patients with recurrent and/or metastatic nasopharyngeal carcinoma that was previously treated with PBT (Chua et al, 2003). Capecitabine was given at doses of $1.250 \mathrm{mg} \mathrm{m}^{-2}$ BID for $1-14$ days every 21 days, observing an ORR of $23.5 \%$ (5.9\% CR and $17.6 \%$ PR), with $52.9 \%$ SD, a median TTP of 4.9 months and median survival of 7.6 months. Thereafter, the same authors reported a retrospective series of 49 patients with similar characteristics, with an ORR of $37 \%$ ( $6 \% \mathrm{RC}, 31 \% \mathrm{RP})$, median TTP of 5 months and median OS of 14 months (Chua et al, 2008). Recently, Ciuleanu et al (2008) have reported another phase II study with capecitabine as rescue treatment (second, third and fourth line) in 23 patients with relapsed nasopharyngeal carcinoma, achieving $48 \%$ objective responses, with mild toxicity.

Our study is the first phase II study in recurrent and/or metastatic HNC specifically assessing the efficacy of capecitabine monotherapy as second-line treatment in patients previously treated with platinum or derivates. The results obtained in the response rates $(24.2 \%)$, median TTP (4.8 months) and median OS (7.3 months) are quite similar to those obtained with most cytostatics, given either alone or in combination, in this subpopulation of patients.

In addition, the regimen has been well tolerated. Most common AEs reported were consistent with those reported in other studies with capecitabine in monotherapy (Walko and Lindley, 2005; Aprile et al, 2009). The most frequent AEs leading to capecitabine dose reductions, cycle delays and treatment discontinuations were non-haematological AEs, namely, palmar-plantar eritrodisestesia, asthenia and mucositis. Particularly, $20 \%$ of patients discontinued treatment because of AEs. It should be noted that our patients represent a particularly fragile subgroup, as most patients had been previously treated with chemoradiotherapy. Generally, these unfit patients are less capable of achieving a good compliance, and are more vulnerable to toxic effects. Probably, this fact explains the high proportion of early withdrawals before completing the minimum established treatment observed in our study; however, it also gives added value to our results when considering capecitabine treatment in these patients. As mentioned before, the study drug was withdrawn early in most cases because of tumour complications, which are characteristic of these patients, but few 
dose delays/reductions were reported because of AEs; therefore, the median relative dose intensity reported was quite high (91\%), despite the discontinuation rate observed.

This regimen is also easy to be administered. Oral administration offers an alternative to the more inconvenient i.v. administration of 5FU. In view of all these characteristics, this treatment may be considered as a suitable therapeutic alternative in a palliative setting. The combination of cytotoxic drugs does not seem to provide more benefits than monotherapy in this sub-population of patients.

Our study was mainly conceived to explore whether capecitabine possesses enough activity when given as monotherapy in HNC patients to be considered for further studies. Therefore, the impact of capecitabine monotherapy on patient's quality of life (QoL) was not assessed. For the same reason, this study did not include translational research assessments. Nevertheless, these facts do not underestimate the clinical relevance of our study.

\section{REFERENCES}

Aprile G, Mazzer M, Moroso S, Puglisi F (2009) Pharmacology and therapeutic efficacy of capecitabine: focus on breast and colorectal cancer. Anticancer Drugs 20: 217-229

Arnedos M, Carbonell X, Del Campo JM, Carulla JM, Urdambidelus A, Galtés S, Baselga J, Trigo J (2002) Phase I study of capecitabine in combination with cisplatin in patients (pts) with advanced and/or metastasic squamous cell carcinoma of head and neck (SCCHN). Ann Oncol 13(Suppl 5): 154-155 (abstract 567P)

Bentzen JD, Hansen HS (2007) Phase II analysis of paclitaxel and capecitabine in the treatment of recurrent or disseminated squamous cell carcinoma of the head and neck region. Head Neck 29: 47-51

Catimel G, Verweij J, Mattijssen V, Hanauske A, Piccart M, Wanders J, Franklin H, Le BN, Clavel M, Kaye SB (1994) Docetaxel (Taxotere): an active drug for the treatment of patients with advanced squamous cell carcinoma of the head and neck. EORTC Early Clinical Trials Group. Ann Oncol 5: 533-537

Chua D, Wei WI, Sham JS, Au GK (2008) Capecitabine monotherapy for recurrent and metastatic nasopharyngeal cancer. Jpn J Clin Oncol 38: $244-249$

Chua DT, Sham JS, Au GK (2003) A phase II study of capecitabine in patients with recurrent and metastatic nasopharyngeal carcinoma pretreated with platinum-based chemotherapy. Oral Oncol 39: 361-366

Ciuleanu E, Irimie A, Ciuleanu TE, Popita V, Todor N, Ghilezan N (2008) Capecitabine as salvage treatment in relapsed nasopharyngeal carcinoma: a phase II study. J BUON 13: 37-42

Forastiere AA, Metch B, Schuller DE, Ensley JF, Hutchins LF, Triozzi P, Kish JA, McClure S, VonFeldt E, Williamson SK (1992) Randomized comparison of cisplatin plus fluorouracil and carboplatin plus fluorouracil versus methotrexate in advanced squamous-cell carcinoma of the head and neck: a Southwest Oncology Group study. J Clin Oncol 10: 1245-1251

Forastiere AA, Shank D, Neuberg D, Taylor SG, DeConti RC, Adams G (1998) Final report of a phase II evaluation of paclitaxel in patients with advanced squamous cell carcinoma of the head and neck: an Eastern Cooperative Oncology Group trial (PA390). Cancer 82: 2270 - 2274

Hitt R, Jimeno A, Rodriguez-Pinilla M, Rodriguez-Peralto JL, Millan JM, Lopez-Martin A, Brandariz A, Pena C, Cortes-Funes H (2004) Phase II trial of cisplatin and capecitabine in patients with squamous cell carcinoma of the head and neck, and correlative study of angiogenic factors. Br J Cancer 91: 2005-2011

Hong WK, Bromer R (1983) Chemotherapy in head and neck cancer. N Engl J Med 308: 75-79
Future studies should take into consideration QoL assessment and the incorporation of a translational research.

The beneficial effects of novel EGFR-targeted drugs such as cetuximab, which have been demonstrated to improve the effectiveness of CT when added to treatment (Vermorken et al, 2008), were not available when this study was designed. Thus, with the limits of a phase II study, data from this study should encourage investigators to use capecitabine in combination with new EGFR inhibitors when designing new studies in HNC patients. In view of the effectiveness demonstrated by capecitabine, this drug should be considered for future combination studies in HNC.

\section{ACKNOWLEDGEMENTS}

This study was supported by Hoffmann-La Roche Inc., Nutley, NJ, USA.

Kim JG, Sohn SK, Kim DH, Baek JH, Jeon SB, Chae YS, Lee KB, Park JS, Sohn JH, Kim JC, Park IK (2005) Phase II study of concurrent chemoradiotherapy with capecitabine and cisplatin in patients with locally advanced squamous cell carcinoma of the head and neck. $\mathrm{Br} J$ Cancer 93: $1117-1121$

Pignon JP, Bourhis J, Domenge C, Designe L (2000) Chemotherapy added to locoregional treatment for head and neck squamous-cell carcinoma: three meta-analyses of updated individual data. MACH-NC Collaborative Group. Meta-Analysis of Chemotherapy on Head and Neck Cancer. Lancet 355: $949-955$

Pivot X, Chamorey E, Guardiola E, Magne N, Thyss A, Otto J, Giroux B, Mouri Z, Schneider M, Milano G (2003) Phase I and pharmacokinetic study of the association of capecitabine-cisplatin in head and neck cancer patients. Ann Oncol 14: $1578-1586$

Sykes AJ, Slevin NJ, MacDougall RH, Ironside JA, Mais KL (2004) Results of a phase I study to determine the maximum tolerated dose of capecitabine when given concurrently with radical radiotherapy in the treatment of squamous cell carcinoma of the head and neck. Radiother Oncol 71: 81-84

Therasse P, Arbuck SG, Eisenhauer EA, Wanders J, Kaplan RS, Rubinstein L, Verweij J, Van GM, van Oosterom AT, Christian MC, Gwyther SG (2000) New guidelines to evaluate the response to treatment in solid tumors. European Organization for Research and Treatment of Cancer, National Cancer Institute of the United States, National Cancer Institute of Canada. J Natl Cancer Inst 92: 205-216

Thomas CY, Read P, Petroni G, Reibel J, Levine PA (2009) Phase I study of capecitabine, carboplatin and intensity-modulated radiation therapy for head and neck cancer. Anticancer Res 29: 2869-2873

Vermorken JB, Mesia R, Rivera F, Remenar E, Kawecki A, Rottey S, Erfan J, Zabolotnyy D, Kienzer HR, Cupissol D, Peyrade F, Benasso M, Vynnychenko I, De RD, Bokemeyer C, Schueler A, Amellal N, Hitt R (2008) Platinum-based chemotherapy plus cetuximab in head and neck cancer. N Engl J Med 359: 1116-1127

Vokes EE, Weichselbaum RR, Lippman SM, Hong WK (1993) Head and neck cancer. $N$ Engl J Med 328: $184-194$

Walko CM, Lindley C (2005) Capecitabine: a review. Clin Ther 27: 23-44

(c) (1) (2) This work is licensed under the Creative Commons C. ${ }_{\text {BY }}$ NC SA Attribution-NonCommercial-Share Alike 3.0 License. To view a copy of this license, visit http://creativecommons.org/ licenses/by-nc-sa/3.0/ 